\title{
Anesthesia for aortic surgery with circulatory arrest in children with Loeys-Dietz syndrome
}

\author{
Yu Zhang, MD, Lei Chen, MD, Hongwen Ji, MD, and Fuxia Yan, MD, Beijing, China
}

\footnotetext{
From the Department of Anesthesiology, State Key Laboratory of Cardiovascular Diseases, National Center for Cardiovascular Diseases, Fuwai Hospital, Peking Union Medical College and Chinese Academy of Medical Sciences, Beijing, China.

No funding was provided for this work.

Disclosures: Authors have nothing to disclose with regard to commercial support.

Received for publication March 22, 2018; revisions received Sept 18, 2018; accepted for publication Sept 27, 2018.

Address for reprints: Fuxia Yan, MD, Department of Anesthesiology, State Key Laboratory of Cardiovascular Diseases, National Center for Cardiovascular Diseases, Fuwai Hospital, Peking Union Medical College and Chinese Academy of Medical Sciences, Beijing 100037, China (E-mail: yanfuxia@sina.com).

J Thorac Cardiovasc Surg 2019;157:e53-5

$0022-5223 / \$ 36.00$

Copyright (C) 2018 Published by Elsevier Inc. on behalf of The American Association for Thoracic Surgery

https://doi.org/10.1016/j.jtcvs.2018.09.122
}

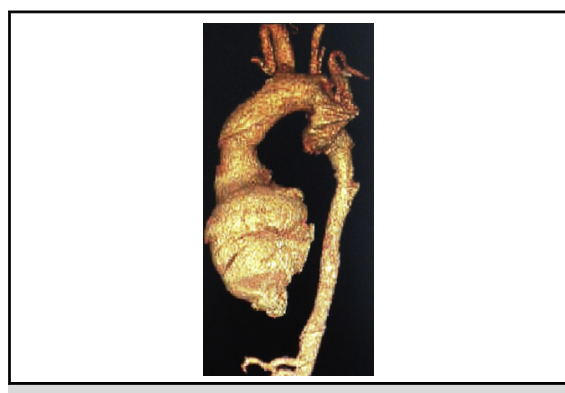

Computed tomographic imaging of aortic dissection.

Central Message

Comprehensive understanding of pathophysiology, detailed preparation, and timely treatment of the emergencies are the key points of anesthesia for aortic surgery in children with Loeys-Dietz syndrome.

See Editorial Commentary page e57.
Loeys-Dietz syndrome (LDS) is characterized by aortic aneurysms and generalized arterial tortuosity, hypertelorism, and a bifid or broad uvula or cleft palate. ${ }^{1}$ Patients with LDS may show rapid progression of aortic dilation, regurgitation, and potential rupture or dissection at young ages and with small aneurysms. The short- and intermediate-term surgical outcomes, however, are favorable. ${ }^{2,3}$ Aortic surgical emergencies in pediatric patients with LDS require meticulous perioperative management to limit morbidity and mortality. We describe here the perioperative management for aortic surgery with deep hypothermic circulatory arrest in 2 pediatric patients with LDS.

\section{CASE PRESENTATIONS}

Case 1

The patient was a 7-year-old boy (body weight, $23 \mathrm{~kg}$ ) with the transforming growth factor- $\beta$ receptor type 1 (TGFBR 1) gene mutation. Computed tomography revealed acute type A aortic dissection, an aortic root diameter of $75 \mathrm{~mm}$, an absolute aortic sinus diameter of $35 \mathrm{~mm}$, and an aortic arch diameter of $33 \mathrm{~mm}$ (Figure 1). Echocardiography demonstrated severe aortic regurgitation, a left ventricular end-diastolic diameter of $68 \mathrm{~mm}$, and an ejection fraction of $55.3 \%$.

Aortic root replacement was performed with a modified Bentall technique with a 21-mm St Jude valve conduit (St Jude Medical, St Paul, Minn). A 26-mm, 4-branch prosthetic graft was used for total arch replacement. The operation time, cardiopulmonary bypass time, aortic crossclamp time, and circulatory arrest time were 285, 163, 93, and 10 minutes, respectively. The lowest nasopharyngeal temperature was $20.7^{\circ} \mathrm{C}$, and the bleeding volume was $300 \mathrm{~mL}$. The patient received fresh-frozen plasma (400 mL), platelets (1 unit), and red blood cells (2 units). The extubation time was 8 hours. The patient was discharged 10 days postoperatively with no complications.

\section{Case 2}

The patient was a 5-year-old boy (body weight, $20 \mathrm{~kg}$ ) with the transforming growth factor- $\beta$ receptor type 2 (TGFBR2) gene mutation. Computed tomography revealed disruption of the aortic isthmus vessel wall, a large thoracic aortic pseudoaneurysm (diameter, $77 \mathrm{~mm}$ ), and depression of the pulmonary trunk and left pulmonary artery (Figure 2). Echocardiography demonstrated a ventricular end-diastolic diameter of $37 \mathrm{~mm}$ and an ejection fraction of $60 \%$.

A 16-mm prosthetic graft was used for replacement after pseudoaneurysm removal. cardiopulmonary bypass was established after cannulation of the femoral artery, femoral vein, and superior vena cava. The operation time, cardiopulmonary bypass time, and aortic crossclamp time under circulatory arrest were 300,145 , and 15 minutes, respectively. 


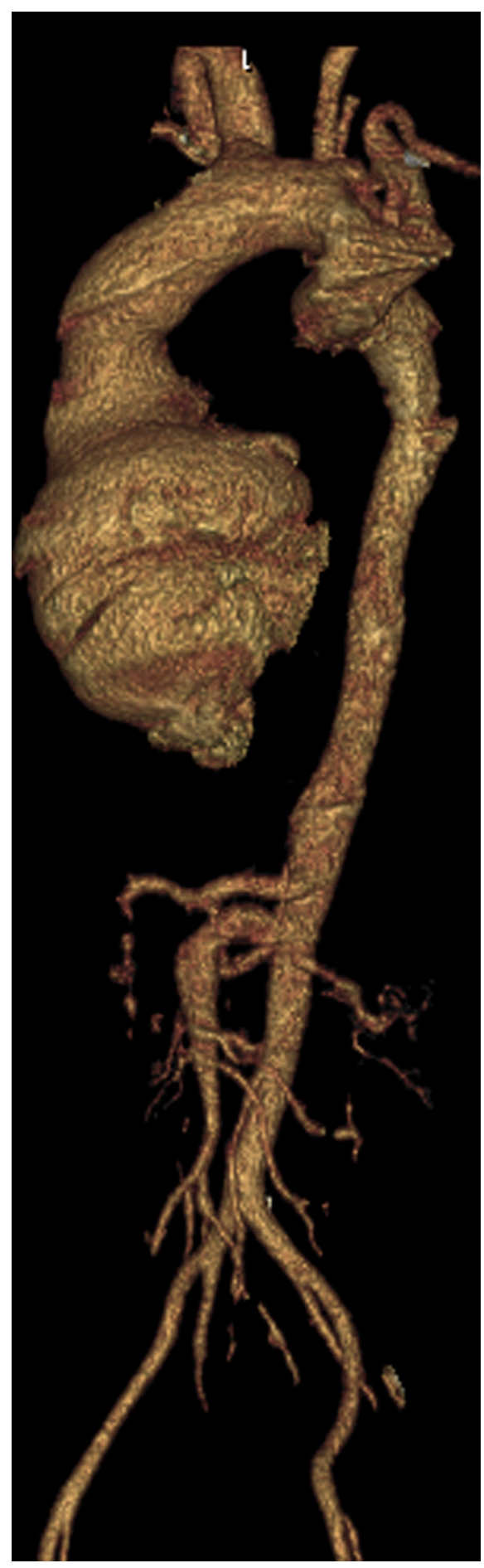

FIGURE 1. Computed tomographic image from case 1. Acute type A aortic dissection caused expansion of the aortic sinus, aortic root, and aortic arch.

The lowest nasopharyngeal temperature was $23.2^{\circ} \mathrm{C}$, and the bleeding volume was $600 \mathrm{~mL}$. The patient received platelets ( 1 unit) and red blood cells ( 2 units). The extubation time was 13 hours. The patient was discharged 9 days postoperatively with no complications.

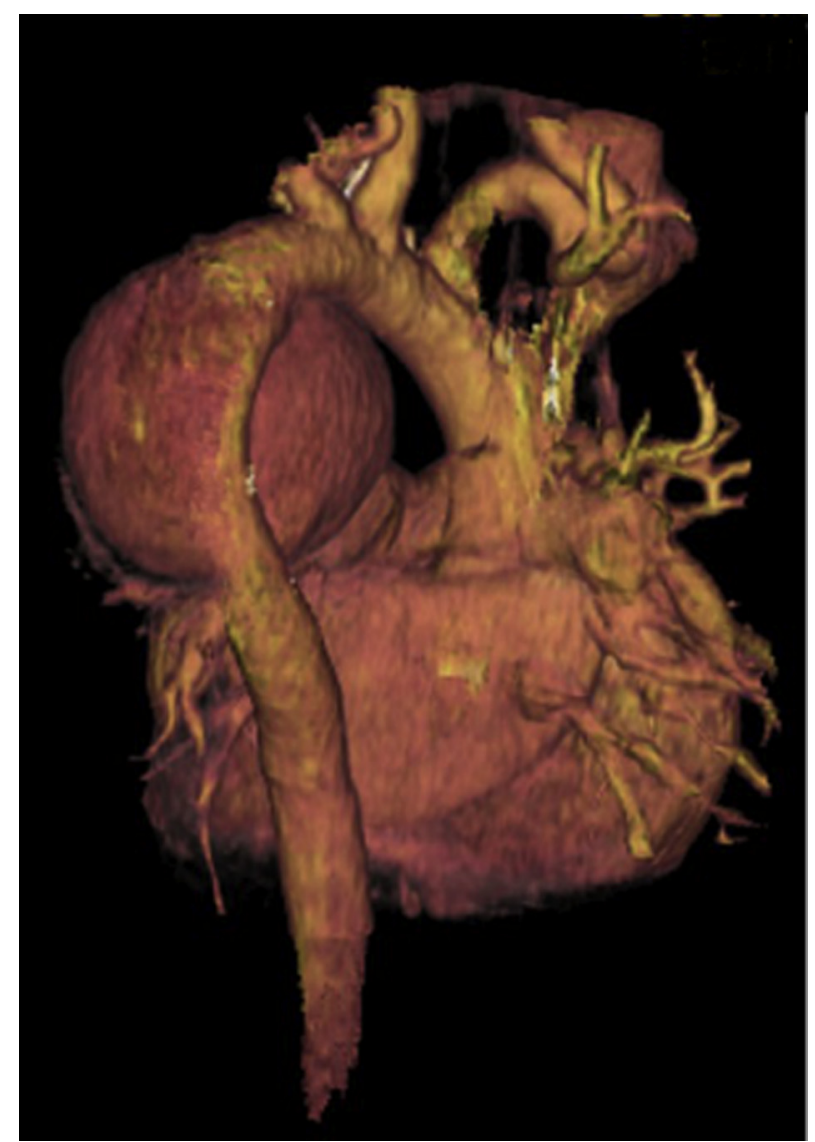

FIGURE 2. Computed tomographic image from case 2. Computed tomography showed disruption of the aortic isthmus vessel wall and a large thoracic aortic pseudoaneurysm.

\section{DISCUSSION}

LDS is characterized by aggressive aortic pathology, typical craniofacial features, joint laxity, and skin abnormalities. Once aortic pathology is diagnosed, surgical repair is needed as soon as possible. ${ }^{1}$ We performed a detailed preoperative anesthetic assessment of the blood pressure, cervical spine, airway, and cardiac status.

In patients with LDS, hypertension can lead to aortic dissection or rupture. Both patients had normal blood pressure and received $2 \mathrm{mg}$ intravenous midazolam before surgery to decrease anxiety. Atenolol and nicardipine were prepared before induction. Minimal cardiovascular responses occurred during intubation and sternal splitting because of the administration of large opioid doses. Because of the patients' increased joint laxity, care was taken to avoid temporomandibular joint subluxation during intubation; this was accomplished without difficulty with a conventional laryngoscope. These management techniques were similar to those reported in a previous case. ${ }^{4}$

In case 2, a standard tracheal tube with an inner diameter of $5.5 \mathrm{~mm}$ was inserted after induction. We then placed a $5 \mathrm{~F}$ endobronchial blocker into the left main stem bronchus 


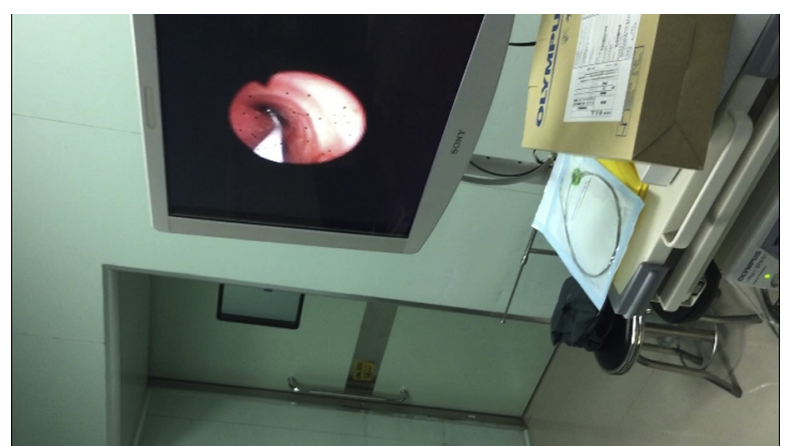

VIDEO 1. Fiberbronchoscopic guidance was used to place an endobronchial blocker into the left main stem bronchus in a pediatric patient with a thoracic aortic pseudoaneurysm. Video available at: https://www.jtcvs. org/article/S0022-5223(18)32852-6/fulltext.

with fiberbronchoscopic guidance to ensure that only rightlung ventilation occurred (Video 1 )..$^{5}$ The respiratory parameters during dual-lung ventilation were as follows: tidal volume of 8 to $10 \mathrm{~mL} / \mathrm{kg}$, respiratory frequency of 18 to 20 breaths/min, and airway pressure of less than $20 \mathrm{~cm} \mathrm{H}_{2} \mathrm{O}$. In contrast, the parameters during right-lung ventilation only were as follows: tidal volume of 6 of $8 \mathrm{~mL} / \mathrm{kg}$, respiratory frequency of 20 to 25 breaths/min, airway pressure of less than $30 \mathrm{~cm} \mathrm{H}_{2} \mathrm{O}$; oxygen saturation by pulse oximetry of more than $90 \%$; and end-tidal carbon dioxide of 35 to $45 \mathrm{~mm} \mathrm{Hg}$. Collapse of the left lung with the endobronchial blocker enabled good exposure of the operative field. The thoracic aortic pseudoaneurysm thus was dissected easily during the prebypass period. On completion of surgery, the endobronchial blocker was removed to facilitate postoperative airway management.

Other necessary perioperative precautions include brain protection, myocardial protection, and blood protection in accordance with the routine management of deep hypothermic circulatory arrest. There is a need for annual follow-up and accumulation of further experience with perioperative management for aortic surgery with deep hypothermic circulatory arrest in pediatric patients with LDS.

\section{References}

1. Schepers D, Tortora G, Morisaki H, MacCarrick G, Lindsay M, Liang D, et al. A mutation update on the LDS-associated genes TGFB2/3 and SMAD2/3. Hum Mutat. 2018;39:621-34.

2. Zanotti G, Vricella L, Cameron D. Thoracic aortic aneurysm syndrome in children. Semin Thorac Cardiovasc Surg Pediatr Card Surg Annu. 2008;11-21.

3. Patel ND, Crawford T, Magruder JT, Alejo DE, Hibino N, Black J, et al. Cardiovascular operations for Loeys-Dietz syndrome: intermediate-term results. $J$ Thorac Cardiovasc Surg. 2017;153:406-12.

4. MacCarrick G, Black JH III, Bowdin S, El-Hamamsy I, FrischmeyerGuerrerio PA, Guerrerio AL, et al. Loeys-Dietz syndrome: a primer for diagnosis and management. Genet Med. 2014;16:576-87.

5. Kamra SK, Jaiswal AA, Garg AK, Mohanty MK. Rigid bronchoscopic placement of Fogarty catheter as a bronchial blocker for one lung isolation and ventilation in infants and children undergoing thoracic surgery: a single institution experience of 27 cases. Indian J Otolaryngol Head Neck Surg. 2017;69: $159-71$. 